\title{
Lateral lamella of the cribriform plate, a keystone landmark: proposal for a novel classification system*
}

\author{
R. Gera'1, F. Mozzanica', A. Karligkiotis², A. Preti², F. Bandi², S. Gallo², \\ A. Schindler ${ }^{3}$, C. Bulgheroni', F. Ottaviani', P. Castelnuovo² \\ Rhinology 56: 65-72, 2018 \\ https://doi.org/10.4193/Rhino17.067 \\ ' Department of Clinical Sciences and Community Health, University of Milan, Milan, Italy \\ *Received for publication: \\ 2 Department of Biotechnology and Life Sciences, University of Insubria, Varese, Italy \\ April 11, 2017 \\ ${ }^{3}$ Department of Biomedical and Clinical Sciences, University of Milan, Milan, Italy \\ Accepted: August 1, 2017
}

\begin{abstract}
Background: The aim of this study is to propose a classification of the angle formed by the lateral lamella of the cribriform plate (LLCP) and the horizontal plane passing through the cribriform plate. In particular, the angle was classified into class I ( $>80$ degrees), class II (45 to 80 degrees, and class III ( $<45$ degrees)
\end{abstract}

Methodology: A total of 190 computed tomography scans were retrospectively reviewed in order to obtain four sets of measurements. 1) depth of the cribriform, 2) angle, 3) length of the LLCP, 4) width of the fovea ethmoidalis. The relationship among these measurements were analyzed.

Results: The angle was significantly correlated with the depth of the cribriform and the length of the fovea, while it was negatively correlated with the length of the LLCP. Significant negative correlation was also found between the length of the LLCP and the width of the fovea.

Conclusions: This angle classification is based on the theoretical risk of iatrogenic injuries, but it could be helpful also in clinical practice by providing indirect information on the thickness of the anterior skull base. As the angle decreases, in fact, the portion of the anterior skull base composed by the LLCP, increases.

Key words: anatomy, radiography, diagnosis, skull base, surgery

\section{Introduction}

Endoscopic sinus surgery (ESS) is a well established approach for the management of sinonasal diseases ${ }^{(1)}$. Although technological advances, proper instrumentation, surgical refinements, use of imaging techniques and better understanding of the regional anatomy have served to make surgeries more secure, ESS is not devoid of complications ${ }^{(2)}$. These latters can be divided into minor ${ }^{(3)}$, and major ones, including cerebrospinal fluid leak (CSF-L) as well as ocular and intracranial injuries ${ }^{(4)}$. Most of these major complications occur during ethmoidectomy and can be related to anatomical variation of the anterior skull base ${ }^{(5)}$.

The anterior skull base is composed by the cribriform plate and by the fovea ethmoidalis. These structures converge at the la- teral lamella of the cribriform plate (LLCP) that delimits laterally the cribriform fossa, a weak area known to be a common site of iatrogenic skull-base injuries due to direct penetration or fracture during middle turbinate manipulation or lack of awareness of instrument placement ${ }^{(6)}$. This fossa, in fact, is the thinnest and the most vulnerable structure of the anterior skull base since the thickness of the LLCP can be only $0.05 \mathrm{~mm}^{(6)}$.

According to Keros' classification ${ }^{(7)}$, it is possible to distinguish between 3 types of cribriform fossa depending on its depth: type 1, 1-3 mm long, type 2, 4-7 mm long and type 3, 8-16 mm long. It has been previously reported that Keros type 3 patients have greatest risk of intracranial entry since the great extension in depth of the cribriform fossa and the thickness of its bone- 
wall make this area more susceptible to iatrogenic skull-base injuries ${ }^{(8)}$. Although Keros' classification is still commonly referred to, several authors highlighted the limitations of this classification system in fully describing the shape of the skull base and predicting the risk of intracranial entry ${ }^{(9-13)}$. In particular, Heaton et al. ${ }^{(9)}$ in their retrospective case-control study reported that a greater slope of the anterior skull base in the coronal plane and a low cribriform height relative to the ethmoidal roof predispose the patient to CSF-L during ESS. It could be speculated that these anatomic characteristics may predispose the injuries of the medial part of the skull base during the dissection of the superior ethmoidal cells, when the surgical instruments are in close proximity to the cribriform plate ${ }^{(12)}$.

Even if the role of olfactory fossa depth has been previously stressed ${ }^{(8,14)}$, only limited data regarding the slope of the anterior skull base and, in particular, of the angulation of the LLCP in the coronal plane are available ${ }^{(12,13)}$. This angle might affect the level of the ethmoidal roof relative to the cribriform plate ${ }^{(12)}$, and consequently could play an important role when approaching the frontal sinus and during the dissection of the more medial ethmoidal cells. The aim of this study is to propose a novel classification of the angulation of the LLCP in the coronal plane based on the theoretical risk of iatrogenic injuries in the thinnest and the most vulnerable structure of the anterior skull base. It must be noted that the evidence of an increased risk for complications related to the angulation of the LLCP is really very limited (only a case control study measured the angle or the fovea ethmoidalis at the level of the posterior ethmoid in a group of patients with CFS leak ${ }^{(9)}$. Therefore, the classification here proposed is strictly hypothetical, although it seems logical and reasonable. In addition, normative data of the angulation of the LLCP in the coronal plane obtained in a large sample of CT scans were provided. Finally, the relationship between this new classification and the Keros' one was assessed.

The importance of this study lies in the fact that this new classification in association with the Keros' one, may lead to a deeper standardization and systematic approach during the preoperative sinus imaging assessment and could be of further help in preventing major complications.

\section{Materials and methods}

\section{Participants}

In this retrospective cohort study, computed tomography (CT) scans obtained in a group of one-hundred-ninety subjects (118 males and 72 females) who present themselves to our department for the investigation of possible or evident sinonasal disease were retrospectively reviewed. All CT scans were performed on high-speed spiral CT scanners using non-contrast axial 1.5-mm sections. Exclusion criteria were: age lower than 18 years, previous trauma, sinonasal tumor, sinonasal polyposis (that precluded the visualization of skull base anatomy), skull-
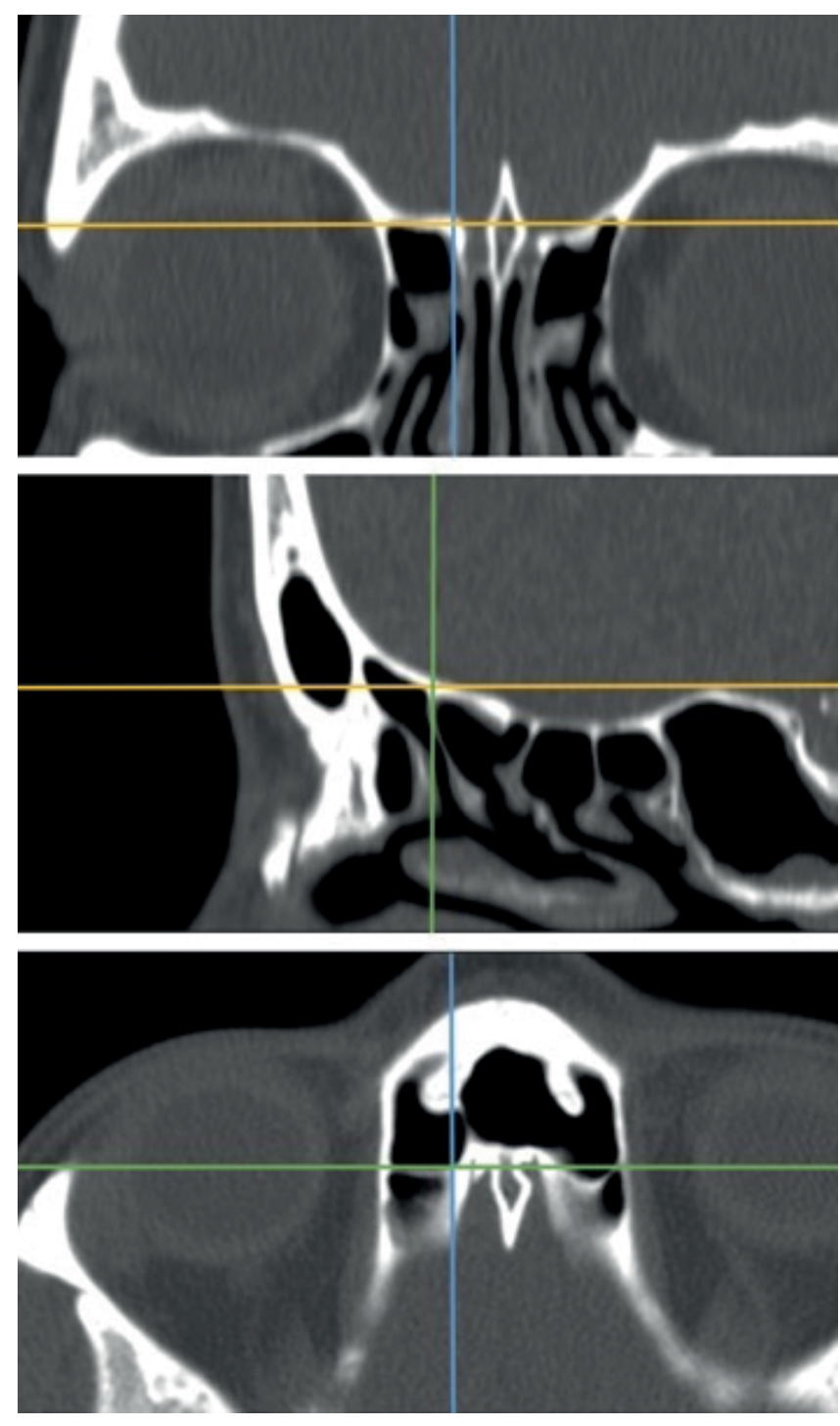

Figure 1. 3-D multi-planar reconstruction (MPR) was used to identify the convergence between the fovea ethmoidalis and the LLCP on the frontal plane at the level of the anterior wall of the first fovea ethmoidalis on the sagittal plane.

base defects, previous ESS and congenital abnormalities of the facial growth. Scans that deviated from a true coronal plane by more than $5^{\circ}$ from the perpendicular plane of the floor of the nose were also excluded. The study was carried out according to the Declaration of Helsinki and it was previously approved by the Institutional Review Board of our hospital.

Similar to Ramakrishnan et al. study ${ }^{(10)}$, high-resolution direct non-contrast axial 1.5-mm sections were analyzed. A specialized computer software "picture archiving and communication system" (PACS) was used for morphological evaluation of the CT scans ${ }^{(15)}$ in order to obtain four sets of measurements. These measurements were taken, using a 3-D multi-planar reconstruction (MPR), at the convergence between the LLCP and the most anterosuperior part of the anterior ethmoidal roof at the level 



Figure 2. Depth of the cribriform measured as the largest vertical height of the olfactory fossa on the coronal plane.
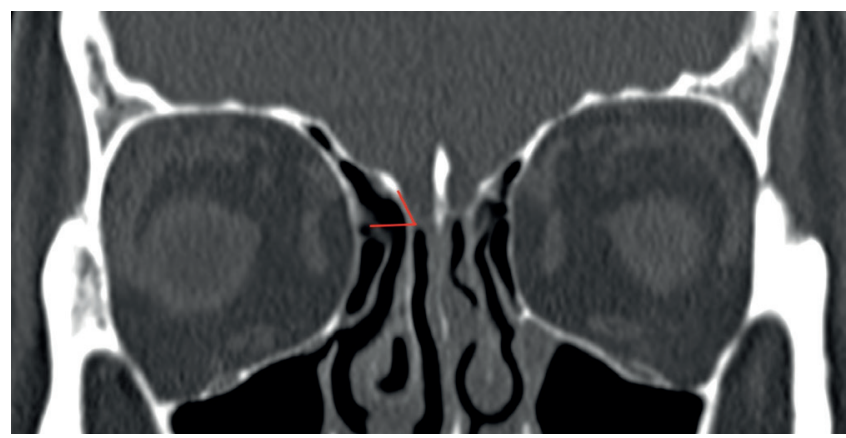

Figure 3. Angle formed by the LLCP and the continuation of the horizontal plane passing through the cribriform plate.

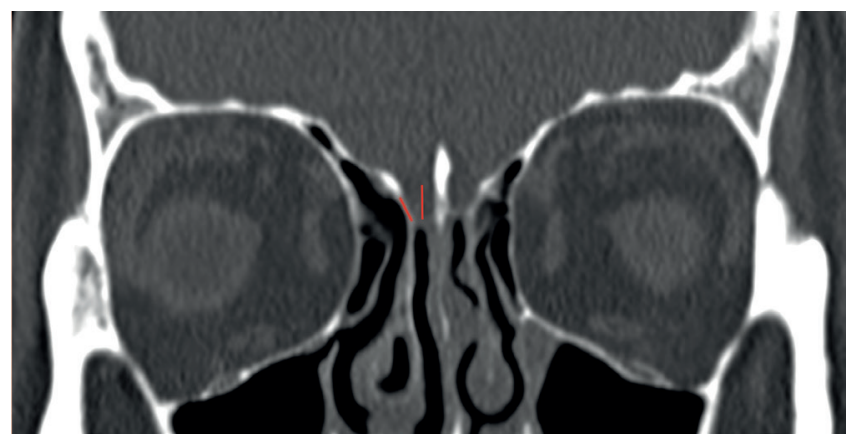

Figure 4. Length of the LLCP.

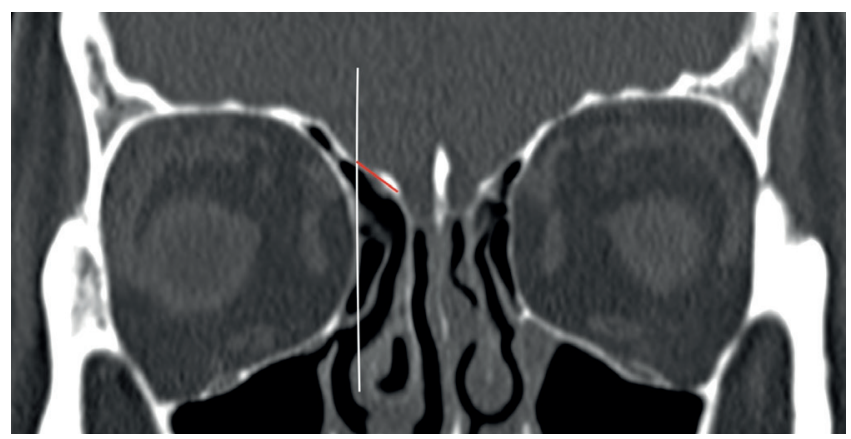

Figure 5. Length of the portion of fovea ethmoidalis included between the LLCP and the vertical line passing at the level of the lamina papiracea. of the posterior margin of the frontal recess ${ }^{(16)}$ on the coronal plane, and at the level of the posterior margin of frontal recess on the sagittal plane (Figure 1). This point was selected since it is well identifiable and represent an important landmark for the dissection of the ethmoidal cells and during the approach to the frontal sinus.

- Depth of the cribriform: the first measurement was the depth of the cribriform plate, measured as the vertical height of the olfactory fossa in the coronal plane on each side (Figure 2). According to Keros' classification system, each CT scan was defined as type 1 (1-3 mm depth), type 2 (4-7 $\mathrm{mm}$ depth) or type 3 (more than $7 \mathrm{~mm}$ depth). Asymmetry in the depth (difference of more than $3 \mathrm{~mm}$ ) between the right and left cribriform plates was also recorded.

- $\quad$ Angle: the second measurement was the angle formed by the LLCP and the continuation of the horizontal plane passing through the cribriform plate (Figure 3). The angle was classified into 3 classes depending on its amplitude and on the hypothetical risk of iatrogenic injuries: class I (> 80 degrees, low risk), class II (45 to 80 degrees, medium risk) and class III ( $<45$ degrees, high risk). The cutoff angles were decided a priori. In particular, the 45 degrees was selected because it is very simple to assess and consequently it should quickly alert the surgeon of potential risk to have the LLCP in a sloped plane. For the same reasons, the other cutoff angle should be 90 degrees. However, preliminary measurements highlighted that no patients scored higher than 90 degrees. The 80 degrees was chosen because on a "quasi" vertical plane and consequently easily to assess.

- Length of the LLCP: the third measurement was the length of the LLCP (Figure 4), considered as the thinnest part of the angulated bony structure connecting the cribriform plate to the ethmoidal roof.

- Width of the fovea: the fourth measurement was the length of the portion of fovea ethmoidalis included between the LLCP and a vertical line passing at the level of the lamina papiracea in the frontal plane reconstruction (Figure 5).

Each CT scan was reviewed by two independent raters (named rater 1 and rater 2), specialized in sinonasal disease and with an experience of at least 20 years in ESS. Correlations between the four sets of measurements were analyzed. In addition, in order to evaluate the inter rater reliability of these measurements, the results obtained by rater 1 and 2 were compared. Finally, in order to evaluate the intra rater reliability, rater 1 analyzed a random sample of 50 CT scans twice, with 2 weeks of interval. During the second evaluation the rater was blind to the results obtained during the first evaluation. 


\section{Statistical analysis}

Statistical tests were performed using the SPSS 21.0 statistical software (SPSS, Inc., Chicago, IL, USA). Mean \pm standard deviation and ranges were calculated for the measurements. The differences in the distribution of the measurements between males and females were evaluated through Student $t$ for continuous variables and through Chi-square test for categorical variables. The degree of association between the angle width, LLCP length, depth of the cribriform plate and length of the portion of fovea ethmoidalis was estimated using Pearson's correlation coefficient. The same test was also used to analyze the inter- and intra-rater reliability of the measurements.

\section{Results}

A total of 190 paranasal sinuses CT scans were retrospectively analyzed. The mean age of the patients included in the study was $43.6 \pm 7.2$ years (range, 19-67 years). One-hundred eighteen patients were males $(62.1 \%)$, the remaining 72 patients were females (37.9\%). The mean age of male patients was 43.2 \pm 8.1 years (range, 19-66 years), while the mean age of female patients was $46.2 \pm 6.9$ years (range, 20-67 years). No differences were found in the distribution of age among males and females on Student $t$ test $(p=0.434)$.

- Depth of the cribriform: the mean depth of the cribriform plate was $5.4 \pm 1.7 \mathrm{~mm}$ (range, 2.4-10.3 $\mathrm{mm}$ ). The mean depth of the cribriform plate in males was $5.6 \pm 1.7 \mathrm{~mm}$ (range, 2.4-10.1 mm) while in females it was $5.2 \pm 1.7 \mathrm{~mm}$ (range, 2.9-10.3 mm). These differences were found not significant on Student $t$ test $(p=0.909)$. The most common anatomic variation was Keros type 2 (64.7\% of cases), followed by Keros type 1 (20\% of cases) and Keros type 3 (15.3\% of cases). No significant differences in the distribution of Keros classification among males and females were found on Chi-square test $(p=0.510)$. Asymmetry in the depth of the cribriform plate was reported in $12.3 \%$ of patients. In $60.2 \%$ of them the height of the fovea ethmoidalis was lower at the right side compared to the left one.

- Angle: the mean degree of the angle formed by the LLCP and the continuation of the horizontal plane passing through the cribriform plate was $71.7 \pm 12.1^{\circ}$ (range $\left.27-89^{\circ}\right)$. The mean angle degree in males was $71.5 \pm 13.7^{\circ}$ (range, $27-87^{\circ}$ ) while in females it was $72.1 \pm 10.7^{\circ}$ (range, $\left.32-89^{\circ}\right)$. These differences were found not significant on Student $t$ test $(p=0.179)$. In $62.7 \%$ of cases, the angle ranged from 45 to 80 degrees (class II, medium risk). In $32.6 \%$ of cases the angle measured more than 80 degrees (class I, low risk) and in $4.7 \%$ of cases the angle measure less than 45 degrees (class III, high risk). No significant differences in the distribution of angle classification among males and females were found on Chi-square test $(p=0.108)$.

- Length of the LLCP: the mean length of the LLCP was $4.7 \pm$
Table 1. Results of the correlation analysis among the ethmoidal measurements.

\begin{tabular}{|c|c|c|c|c|}
\hline & $\begin{array}{l}\text { Depth of } \\
\text { the } \\
\text { cribriform }\end{array}$ & LLCP & Angle & $\begin{array}{l}\text { Width of } \\
\text { the fovea }\end{array}$ \\
\hline $\begin{array}{l}\text { Depth of the cribri- } \\
\text { form }\end{array}$ & 1 & $0.338^{*}$ & $0.553^{* *}$ & $0.385^{*}$ \\
\hline LLCP & $0.338^{*}$ & 1 & $-0.397^{*}$ & $-0.342^{*}$ \\
\hline Angle & $0.553^{* *}$ & $-0.397^{*}$ & 1 & $0.336^{*}$ \\
\hline Width of the fovea & $0.385^{*}$ & $-0.342^{*}$ & $0.336^{*}$ & 1 \\
\hline
\end{tabular}

Table 2. Results of inter- and intra-rater reliability analysis.

\begin{tabular}{|lcc|}
\hline & Intra-rater & Inter-rater \\
\hline Depth of the cribriform & 0.85 & 0.82 \\
\hline LLCP & 0.90 & 0.87 \\
\hline Angle & 0.92 & 0.90 \\
\hline Width of the fovea & 0.89 & 0.86 \\
\hline
\end{tabular}

$1.4 \mathrm{~mm}$ (range, 2.9-10.7 mm). The mean length of LLCP in males was $5.0 \pm 1.5 \mathrm{~mm}$ (range, 2.9-10.7 $\mathrm{mm}$ ) while in females it was $4.5 \pm 1.7$ (range, 3.1-10.4 mm). These differences were found not significant on Student $t$ test $(p=0.097)$.

- Width of the fovea: the mean length of the fovea ethmoidalis was $8.8 \pm 2.3 \mathrm{~mm}$ (range, 2.4-16.7 $\mathrm{mm}$ ). The mean length in males was $8.8 \pm 2.4 \mathrm{~mm}$ (range, $2.4-16.7 \mathrm{~mm}$ ) while in females it was $8.9 \pm 2.2$ (range, 2.5-16.4 mm). These differences were found not significant on Student $t$ test $(p$ $=0.276$ ).

The results of the correlation analysis are reported in Table 1. Figures 6-8 reported the scatterplots showing the relation between the angle formed by the LLCP and the continuation of the horizontal plane passing through the cribriform plate and the others parameters. The angle was positively correlated with the depth of the cribriform and the width of the fovea, while it was negatively correlated with the length of the LLCP. The length of the LLCP was negatively correlated with the angle and with the length of the fovea. In particular, class I patients (angle width more than 80 degrees), scored higher in the width of the fovea and in the depth of the cribriform. On the contrary, class III patients (angle width less than 45 degrees) presented higher values in LLCP length.

The results of intra- and inter-rater reliability analysis are reported in Table 2. The intra- and inter-rater reliability was higher than $r=0.82$ for all the 4 measurements and reached the value of $r=0.92, p=0.022$ and $r=0.90, p=0.32$ for the intra- and inter-rater reliability of the measurement of the angle between 


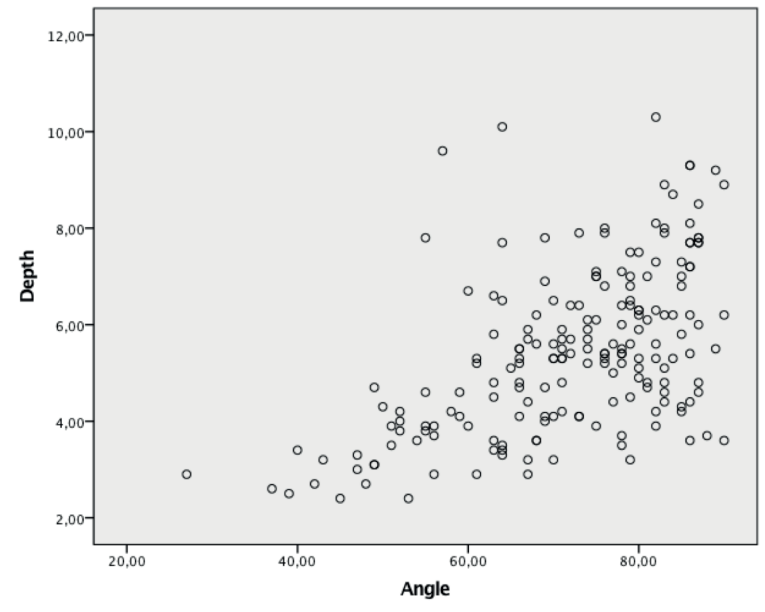

Figure 6. Scatterplots showing the relation between the depth of the cribriform and the degree of the angle formed by the LLCP and the continuation of the horizontal plane.

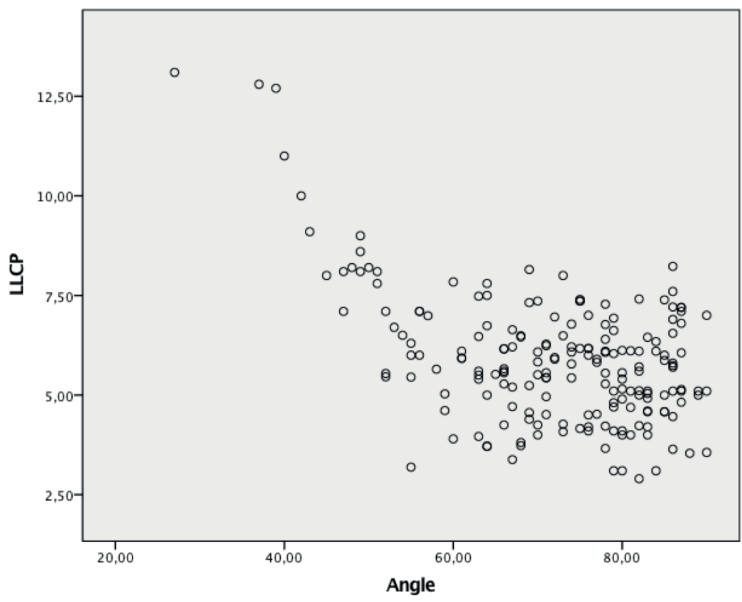

Figure 7. Scatterplots showing the relation between the length of the LLCP the degree of the angle formed by the LLCP and the continuation of the horizontal plane.

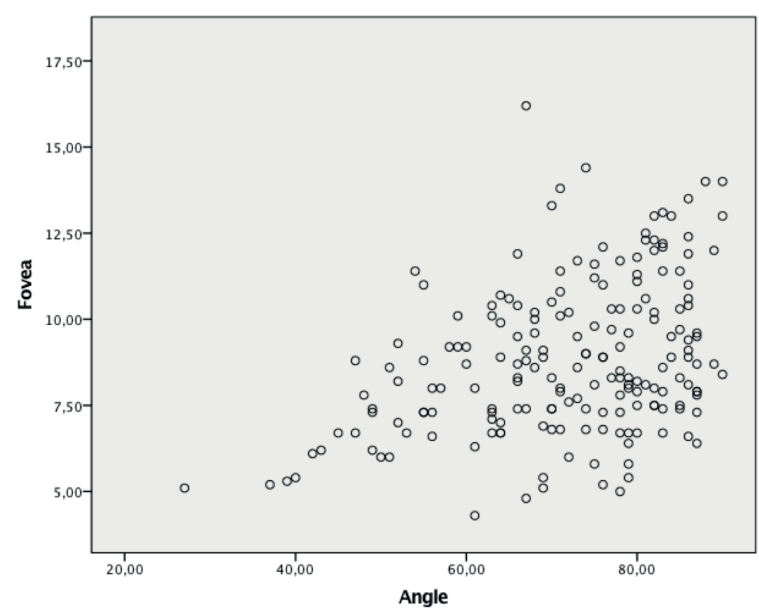

Figure 8. Scatterplots showing the relation between the length of the portion of fovea ethmoidalis included between the LLCP and the vertical line passing at the level of the lamina papiracea and the degree of the angle formed by the LLCP and the continuation of the horizontal plane. the LLCP and the cribriform plate respectively.

The distribution of the angle classes and Keros types among the 190 enrolled patients is reported in Table 3. The majority of patients were classified as Keros type 2 and angle class II, followed by patients classified as Keros type 1 and angle class II and patients classified as Keros type 3 and angle class II.

\section{Discussion}

In this study the slope of the anterior skull base in the coronal plane and its relation with the Keros' classification was analyzed in a large group of patients complaining sinonasal disease. The underlining hypothesis is that a more pronounced slope of the anterior skull base on the coronal plane, may predispose to injuries of the medial part of the skull base during the dissection of the more medial ethmoidal cells and during the approach to the frontal sinus. In fact, during these surgical steps the instruments are in close proximity to the LLCP and the dissection is carried from posterior to anterior; thus it is possible that a more pronounced ethmoidal roof slope may predispose surgeons to inadvertent skull base violation. Consequently, a deeper understanding of this anatomical region is needed in order to reduce the risk of complications during ESS. To the best of our knowledge only few studies analyzed the slope of the anterior skull base $\mathrm{e}^{(10,12,13)}$ but scarce information is available regarding its relationship with other measurements of the ethmoid analyzed through computed tomography. In addition, in none of the previous studies the measurement of the degree of the ethmoidal roof slope in the coronal plane was performed. Only Heaton et al. ${ }^{(9)}$ compared the slope of the skull base on the coronal plane in CSF-leak patients and in a control group and concluded that CSF-leak patients have a larger slope of the skull base than those of the control group. However, no information regarding the degree of the angle of the skull base were provided. In the present study, data on the degree of the angle formed by the LLCP and the continuation of the horizontal plane passing through the cribriform plate in a population complaining sinonasal disease were provided and a new 3-points classification of the slope of the anterior skull base in the coronal plane was proposed. In particular, we propose to classify the angle into 3 classes, depending on its amplitude and on the theoretical risk of iatrogenic injuries: class I (> 80 degrees, low risk), class II (45 to 80 degrees, medium risk) and class III ( $<45$ degrees, high risk). In the present series the large majority of the patients were classified as class I (low risk) and II (moderate risk), while only 4.6\% of patients were classified as at high risk for iatrogenic injuries. Among the patients classified as class III angle, 3.6\% demonstrated a Keros type 1, 0.5\% demonstrated a Keros type 2 and $0.5 \%$ demonstrated a Keros type 3 on the right side, whereas $4.2 \%$ demonstrated a Keros type 1, 0.5\% demonstrated a Keros type 2 and $0 \%$ demonstrated a Keros type 3 on the left side (Table 3). It might be consequently speculated that a significant percentage 
Table 3. Distribution of Keros classification and angle formed by the LLCP and the continuation of the horizontal plane passing through the cribriform plate.

\begin{tabular}{|c|c|c|c|c|c|c|}
\hline & \multirow{2}{*}{$\begin{array}{c}\text { Right (\%) } \\
\text { Class I }\end{array}$} & \multicolumn{5}{|c|}{ Left (\%) } \\
\hline & & Class II & Class III & Class I & Class II & Class III \\
\hline Keros 1 & 3.2 & 13.1 & 3.6 & 3.2 & 12.6 & 4.2 \\
\hline Keros 2 & 20.3 & 43.8 & 0.5 & 21.1 & 43.2 & 0.5 \\
\hline Keros 3 & 8.5 & 6.5 & 0.5 & 10.1 & 6.3 & - \\
\hline Total & 32 & 63.4 & 4.6 & 33.2 & 62.1 & 4.7 \\
\hline
\end{tabular}

Table 4. Distribution of Keros' classification in previous studies.

\begin{tabular}{|lcccc|}
\hline \multicolumn{1}{|c}{ Author } & $\begin{array}{c}N^{\circ} \\
\text { patients }\end{array}$ & \multicolumn{3}{c}{ Keros' classification } \\
\hline Basak et al. ${ }^{(15)}$ & & 1 & $\mathbf{2}$ & 3 \\
\hline Jang et al. ${ }^{(16)}$ & 61 & $9 \%$ & $53 \%$ & $38 \%$ \\
\hline Anderhuber et al. ${ }^{(17)}$ & 205 & $30.5 \%$ & $69.5 \%$ & - \\
\hline Souza et al. ${ }^{(18)}$ & 272 & $14.2 \%$ & $70.6 \%$ & $15.2 \%$ \\
\hline Sahin et al. ${ }^{(19)}$ & 200 & $26.3 \%$ & $73.3 \%$ & $0.5 \%$ \\
\hline Guler et al. ${ }^{(20)}$ & 100 & $10 \%$ & $61 \%$ & $29 \%$ \\
\hline Remarkrishan et al. ${ }^{(10)}$ & 200 & $42 \%$ & $50 \%$ & $8 \%$ \\
\hline Peber et al. ${ }^{(21)}$ & 109 & $81.6 \%$ & $17.9 \%$ & $0.5 \%$ \\
\hline
\end{tabular}

Keros type 2 was more common in men, while type 1 was commoner in women. However, also in Remarkrishan et al. ${ }^{(10)}$ study no differences in the distribution of olfactory fossa height was found among males and females.

In the present study, asymmetry in the depth of the cribriform was reported in $12.3 \%$ of patients and in the majority of the cases the height of the fovea ethmoidalis was lower at the right side compared with the left side. These findings are similar to those of Lebowitz et al. ${ }^{(12)}$, who analyzed 200 CT scans, and reported an asymmetry of the ethmoidal roof in $9.5 \%$ with a fovea ethmoidalis lower on the right side in the majority of cases. Dessi et al. ${ }^{(26)}$, found the right fovea ethmoidalis lower than the left fovea ethmoidalis in patients with olfactory fossa asymmetry. Also Cumberworth et al. ${ }^{(27)}$ who analyzed through postal questionnaires (completed by members of the British Association of Otolaryngologist) the incidence of major complications during ESS, reported higher incidence of CSF leak on the right side, possibly related to asymmetry in the depth of the cribriform.

The high frequency of ethmoidal roof asymmetry further suggests careful preoperative review of the anatomical variations with CT scan in order to prevent the complications.

A significant positive correlation between the depth of the cribriform fossa, the length of LLCP and the degree of the angle between the LLCP and the cribriform plate was found. This agrees with the findings of Elwany et al. ${ }^{(1)}$ who reported that the depth of the olfactory fossa depends upon the length and angulation of the lateral lamella. Also Souza et al. ${ }^{(19)}$ observed that the ethmoidal roof asymmetry was related to the angulation of the lateral lamella. It must be noted that the length of the LLCP was negatively correlated with the length of the fovea ethmoidalis and the degree of the angle. These data suggest that subjects with a more pronounced slope of the anterior skull base on the coronal plane could have a longer LLCP and a shorter fovea ethmoidalis, than subjects with a less pronounced slope of the anterior skull base. It is consequently possible to speculate that the degree of the angle formed by the LLCP and the continuation of the horizontal plane passing through the cribriform plate 
could provide indirect information regarding the thickness of the anterior skull base since, as the angle decreases, the portion of the anterior skull base composed by the LLCP, increases. Interestingly, the correlation between the LLCP and the depth of the cribriform was the poorest of all, in addition, the depth of the cribriform appeared longer than the LLCP. This datum could be related to several factors. First of all, in this study we measured only the length of the LLCP (considered as the thinnest part of the angulated bony structure connecting the cribriform plate to the ethmoidal roof) and is consequently not surprising that the depth of the cribriform could be longer than the LLCP. Moreover, the poor correlation between the depth of the cribriform and the length of the LLCP could be also related to the angulation of the latter (for example in cases where the LLCP is long but very angulated on the coronal plane and consequently the depth of the cribriform was considered small).

Information regarding the intra- and inter-rater reliability of ethmoidal measurements were provided for the first time. Both intra- and inter-rater reliability were high for all the 4 measurements suggesting a good reproducibility over time. This data might be related to the anatomical landmarks used for the measurements. All the measurements, in fact, were performed at the convergence between the LLCP and the most anterosuperior part of the anterior ethmoidal roof at the level of the posterior margin of the frontal recess ${ }^{(17)}$ on the coronal plane, and at the level of the posterior margin of frontal recess on the sagittal plane. Consequently, it is possible that the use of a well identifiable landmarks as well as of MPR could have improved the reliability of the measurements.

\section{Conclusion}

In conclusion, in the preoperative evaluation of a patient with sinonasal disease, careful evaluation of the CT scan is manda- tory. In the past years, the height of the ethmoidal skull base was considered as the most important factor for identification of high-risk anatomic situations within the ethmoid. However, the presence of significant correlations among different ethmoidal measurements highlights the importance of assessing more than just the height of the ethmoidal skull base. In particular, the evaluation of the angle formed by the LLCP and the continuation of the horizontal plane passing through the ethmoidal plate could provide indirect information regarding the thickness of the anterior skull base, which is, in the opinion of the Authors, crucial before surgery. A better analysis of the convergence between the frontal sinus and the ethmoid on the skull base could assure a 'safer' surgical approach to this area. We believe that the application of the classification system here proposed could be consequently useful in the preoperative assessment of sinus imaging in order to prevent major complications. However, future studies including patients with CSF leak caused by injuries at the level of LLCP occurred during ESS procedures are required to validate the classification here proposed.

\section{Authorship contribution}

RG, PC, AS, FO: Substantial contributions to the conception or design of the work, revising it critically for important intellectual content, final approval of the version to be published.

AP, FB, SG: Substantial contributions to the acquisition of data for the work, revising it critically for important intellectual content and final approval of the version to be published.

FM, AK: Substantial contributions to the analysis and interpretation of data for the work. Drafting the work. Final approval of the version to be published.

\section{Conflict of interest}

None of the authors has any conflict of interest to declare.

\section{References}

1. Elwany S, Medanni A, Eid M, et al Radiological observations on the olfactory fossa and ethmoid roof. J Laryngol Otol. 2010; 124: 1251-1256.

2. Bayram M, Sirikci A, Bayaznt Y. Important anatomic variations of the sinonasal anatomy in light of endoscopic surgery: a pictorial review. Eur Radiol. 2001; 11: 1991-1997.

3. Alassawi S, Omar R, Rahmat K, Alli K. Radiological analysis of the ethmoid roof in the Malaysian population. Auris Nasus Larynx. 2012; 39: 393-396.

4. Ulualp SO. Complications of endoscopic sinus surgery: appropriate management of complications. Curr Opin Otolaryngol Head Neck Surg. 2008; 16: 252-259.

5. Dessi P, Castro F, Triglia JM, et al. Major complications of the sinus surgery: a review of 1192 procedures. J Laryngol Otol. 1985; 108: 212-215.
6. Ohnishi T, Yanigasawa E. Lateral lamella of the cribriform plate an important high-risk area in endoscopic sinus surgery. Ear Nose Throat J. 1995; 74; 688-690.

7. Keros P. On the pratical value of differences in the level of the lamina cribrosa of the ethmoid. Laryngorhinootologie. 1962; 41: 808-813.

8. Kainz J, Stammberger $\mathrm{H}$. The roof of the anterior ethmoid: a locus minoris resistentiae in the skull base. Laryngorhinootologie. 1988; 66; 142-149.

9. Heaton CM, Goldberg AN, Pletcher SD, Glastonbury CM. Sinus Anatomy Associated With Inadvertent Cerebrospinal Fluid Leak During Functional Endoscopic Sinus Surgery. Laryngoscope. 2012; 122: 14461449.

10. Ramakrishnan VR, Suh JD, Kennedy DW. Ethmoid skull-base height: a clinically relevant method of evaluation. Int Forum
Allergy Rhinology. 2011; 11: 396-400.

11. Lee JC, Song YJ, Chung YS, et al. Height and shape of the skull base as risk factors for skull base penetration during endoscopic sinus surgery. Ann Otol Rhinol Laryngol. 2007; 116: 199-205.

12. Lebowitz RA, Terk A, Jacobs JB, et al. Asymmetry of the ethmoid roof: analysis using coronal computed tomography. Laryngoscope. 2001; 111: 2122-2124.

13. Meyers RM, Valvassori G. Interpretation of anatomic variations of computed tomography scans of the sinuses: a surgeon's perspective. Laryngoscope. 1998; 108: 422-425.

14. Savvateeva DM, Guldner C, Murthum T, et al. Digital volume tomography (DVT) measurements of the olfactory cleft and olfactory fossa. Acta Oto-Laryngologica. 2010; 130: 398-404.

15. Basak S, Akdilli A, Karaman CZ. Assessment of some important anatomical variations 
and dangerous areas of the paranasal sinus by computed tomography in children. Int Pediatr Otolaryngol. 2005; 55: 81-90.

16. Lund VJ, Stammberger H, Fokkens WJ, et al. European position paper on the anatomical terminology of the internal nose and paranasal sinuses. Rhinol Suppl. 2014 Mar;(24):1-34

17. Jang YJ, Park HM, Kin HG. The rediographic incidence of bony defects in the lateral lamella of the cribriform plate. Clin Otolaryngol Allied Sci. 1999; 24: 440-442.

18. Anderhuber W, Walch C, Fock C. Configuration of the ethmoid roof in children 0-14 years of age. Laryngorhinootologie. 2001; 80: 509-511.

19. Souza SA, Souza MM, Idagawa M, et al. Computed tomography assessment of the ethmoid roof: a relevant region at risk in endoscopic sinus surgery. Radiol Bras. 2008, 41: 143-147.

20. Sahin C, Yilmaz Y, Titiz A, et al. Turk Toplumunda etmoid cati ve kafa taban analizi. KBB ve BBC dergisi. 2007; 15: 1-6.

21. Guler C, Uysal IO, Polat K, et al. Analysis of the ethmoid roof and skull base with coronal section paranasal sinus computed tomography. J Craniof Surg. 2012; 23: 14601464.

22. Peber JEL, Cabato MSD, Villarta RL, et al Radiographic analysis of the ethmoid roof based on Keros classification among Filipinos. Philipp J Otolaryngol Head Neck Surg. 2008; 23: 15-19.

23. Erdem G1, Erdem T, Miman MC, Ozturan O A radiological anatomic study of the cribriform plate compared with constant structures. Rhinology. 2004; 42: 225-229.

24. Meloni F, Mini R, Rovasio S, et al. Anatomic variations of surgical importance in ethmoid labyrinth and sphenoid sinus. A study of radiologic anatomy. Surg Radiol Anat. 1992; 14: 65-70.

25. Badia L, Lund VJ, Wei W, Ho WK. Ethnic variation in sinonasal anatomy on CT-scanning. Rhinology. 2005; 43: 210-214.
26. Dessi P, Moulin G, Triglia JM, et al. Difference in the height of the right and left ethmoida roofs: a possible risk factor for ethmoidal surgery. Prospective study of 150 CT scans. J Laryngol Otol. 1994; 108: 261-262.

Francesco Mozzanica

Division of Otorhinolaryngology

Department of Clinical Sciences and

Community Health

University of Milan

Italy

Tel: +39-340-466 2852

E-mail:

Francesco.mozzanica@gmail.com 